\title{
Inquiry Learning with Videodiscs and Computers: An Innovative Teaching Method for Horticulture Courses
}

\author{
John W. White, David J. Beattie, and Perry Kubek \\ Department of Horticulture, Pennsylvania State University, University Park, PA 16802
}

\begin{abstract}
Videodiscs and inquiry-learning computer courses are well-adapted to the visual world of horticulture. Videodiscs look like compact audio discs, but contain audio and visual images. The visual images can be individual slides or video tape segments. The images can be displayed on a video monitor as still frames or as video series with almost instantaneous display of any of 55,000 images, with or without audio. Each of the 55,000 images can be linked by computer software and displayed at any time for an inquiry-learning, self-paced course.
\end{abstract}

Inquiry learning is an educational process by which students are encouraged to interact to a series of questions and guided prompts from text, audio, and visual information on a computer and videodiscs. Discovery learning focuses more on stimulating students to search beyond the guided instructions to access information outside the lesson plan, skip over or rearrange the order of inquiry, or search for details that are not part of the course requirement. Inquiry and discovery learning can be accomplished without the direct supervision of a teacher.

Variations in appearance of plants at various stages of growth and among the same taxa grown with different environments makes the use of live plant material very valuable in teaching horticulture, especially plant identification and taxonomy. However, the range of live plants is limited by available space and funds. Consequently, many courses are taught with slides, especially when offered as distance education or continuing education courses. The number of slides, thus the number of views of each taxon that can be shown in a given period, and the number of comparisons that can be made among various taxa, often is limited. The constraints of having live plant material available in season, limited time in lecture or laboratory to show slides, and limited comparisons of images per taxon are reduced by the use of inquiry learning using videodiscs and computers.

Introductory courses in horticulture, which need to be taught at community colleges or branch campuses where faculty with ad-

Received for publication 12 Oct. 1988. Contribution no. 128. Dept. of Horticulture. Authorized for publication as Paper no. 7994 in the Journal Series of the Pennsylvania Agricultural Experiment Station. The cost of publishing this paper was defrayed in part by the payment of page charges. Under postal regulations, this paper therefore must be hereby marked advertisement solely to indicate this fact. vanced degrees in horticulture may not be available, are another example where a teacher would benefit by having videodiscs and inquiry-learning software available to supplement traditional lectures and laboratories.

\section{THE PROBLEMS}

The exposure of three generations of students to first movies and television, then videotapes and now to videodiscs, challenges teachers to go beyond the traditional lecture format classroom teaching methods (Solomon, 1983). Today, some students expect learning to be entertaining as well as informative.

Large classes and specialized subject matter bring together students with great differences in basic knowledge, learning speed, interest, and response to teaching methods. Guskey and Easton (1988) believe that required introductory courses with large enrollments of heterogeneous students are the most difficult to teach.

The increasing cost of education has made it more difficult for adults who need additional training or retraining to attend a branch campus or travel to a main university campus, the only location where many courses are offered. Greater opportunities are needed for students to reenter educational institutions for updating and retraining, using curriculum material that is tailored to an individual's needs and pace and that upgrades occupational knowledge and skills.

\section{What wc propose to do about the} problems

We propose the use of a network of satellite telecommunication systems, videotapes, videodiscs, traveling lecturers, county extension specialists, and on-site coordinators to provide a more cost-effective and efficient delivery system of required introductory courses. The expected audience will be associate and baccalaureate degree students at community colleges and at satellite campuses and adults (continuing education), with or without degrees, seeking retraining or first degrees.

The basic concept is to use content experts residing at the main university campus more efficiently, through the use of advanced technology delivery systems. Penn State is already using a satellite telecommunications system for transmitting lectures from the University Park (UP) campus to many commonwealth $(\mathrm{CW})$ campuses. County extension offices already are linked by a Macintosh computer network to each other and to the
UP campus. Videotapes and videodiscs would be set up as computer-based, inquiry-learning lessons for self-paced instruction in learning centers at each CW campus and in county extension offices. Traveling lecturers would train on-site coordinators who would advise students, monitor learning progress, proctor exams, and help organize field trips with the assistance of local businessmen and county extension personnel. Content experts at the main campus would work with media specialists from the Division of Instructional Services in structuring lessons for satellite telecommunications and for interactive computer videodisc delivery systems. Extension staff, master gardeners, and commercial personnel could provide workshops and field trips as supplements to the satellite and inquiry course material.

A pilot study will be developed using a series of horticulture courses. Horticulture has a wide appeal to the general public. It provides ties with our basic concerns for health and nutrition, conservation of natural resources and environmental protection, as well as enhancing the quality of life through the visual arts of landscaping and flower arranging. Gardening is one of the top leisure-hour pastimes.

Unique characteristics of videodiscs. Some unique characteristics of interactive videodiscs that make them a promising medium for teaching are:

a) They provide many presentation forms (i.e., rich visuals and audio sequences, graphics, and overlay graphics) (Bunderson, 1980; Cohen, 1984).

b) Interactive discovery or inquiry requires user participation and control and enhances learning, problem-solving, and decision-making skills. Learners can control their own learning sequence, content, forms of representation, speed of presentation (slow motion, fast motion, or still frames), and overall pace (Merrill, 1980; Mellin, 1987).

c) They provide immediate and appropriate feedback and reinforcement because material can be presented according to the needs and ability level of individual learners. Management and record-keeping capabilities of the system allow for cumulative records to be kept, which encourages individualization (O'Shea and Self, 1983).

d) In addition to vast storage capacity, videodiscs provide multiple ways of accessing information, including opportunities for repetitious practice, multiple examples, different problem types, and simulated experiences (Gindele and Gindele, 1984; Smith and Jones, 
1986; Hannaway et al., 1988).

e) Videodiscs can add interest, and stimulate enthusiasm and motivation due to the intrinsic appeal of visual images, stimulations, feedback, individualized instruction, and games (Malone, 1981; Russell et al., 1985).

Behavioral and cognitive learning and videodisc utility. With the aid of videodiscs, learners may conveniently: a) Observe and review demonstrations, procedures, or operations through the use of videotaped sequences taken at commercial businesses; b) Execute otherwise hazardous procedures and operations through guided responses, games, and simulations; c) Memorize and practice recall in response to photographs, graphics, or line drawings; d) Apply concepts, principles, and procedures to visual examples; e) Classify visual presentations according to categories (plant families); f) Compare drawings and photographs with verbal descriptions and with live plants; and g) Diagnose problems, malfunction or disease, and propose remedies using expert and artificial intelligence systems.

Advantages of interactive video instruction. The more participating and self-paced learning environment offered by interactive videodisc instruction should provide not only a quantitatively, but also a qualitatively, different kind of learning experience (Bunderson et al., 1984). This type of learning experience assumes that education involves more than just sorting and storing facts, replacing it with the assumption that "educated people are really problem solvers and decision makers, capable of handling whatif assumptions, complex models and variable outcomes" (Emmett, 1984, p. 62).

In discovery learning, visual images have a very different function than when used as a part of a traditional lecture. Pictures typically are the initial means by which learners inductively explore and extrapolate for themselves the answers to their questions, reasoning and testing from the particular to the general. A good illustration of this process was demonstrated in the videodisc lesson "How Does Light Affect Plants" (Mellin, 1987). Videodisc systems have the potential capacity to enhance the design of both expository and discovery-learning strategies. It is possible to provide learners with immediate access to an abundant choice of visual material for illustrative purposes or for inductive analysis (Young and Tosti, 1981). The range of visual material can include photocollections of scientific specimens; historical documents; maps; film or videotapes of natural processes, disasters, and accidents; interpersonal interaction; and historical events. The engineering videodisc lesson on why the Tacoma Bridge collapsed is another good example of guided discovery learning (Fuller et al., 1982). Interactive video provides several unique advantages for postsecondary or continuing education material offered by the cooperative extension service (Kay and Tate, 1986). Those identified by Deter (unpublished data) include: a) Costeffective delivery; b) Extending the special- ist's outreach ability; c) Potential for increasing the number of clientele served; d) Delivery of expertise to locations currently out of reach; e) Maintain and increase program delivery in times of limited resources; f) Provide information delivery at times and places not currently served; g) Increase the rate of knowledge retention; h) Obtain statistics on student's needs through the use of built-in evaluations; and i) Permits teachers to focus more clearly on being managers of learners.

Bunderson et al. (1984) found greater learning gains for students exposed to videodisc than for those taught by a traditional method. Bunderson et al. (1984) and Clark (1984) observed that the total time in instruction for videodisc students was reduced by $25 \%$ or more when compared to traditional educational methods. In addition, Bunderson found that outside study time for students learning from videodiscs decreased by $41 \%$ when compared with a classroom group. When students complete the course requirements in less than the allotted term or semester time, they can be encouraged to explore suggested information in greater depth for extra credit or begin another course.

Most people can learn any subject matter and develop skills when given enough time and can use repetition to learn more difficult or complex material (Weiss, 1986, Wollenberg et al., 1985). It is expensive to provide this type of individualized instruction using teachers with advanced degrees. Paraprofessionals and master gardeners can guide students in using combinations of commercial businesses, botanic gardens and other local resources, while using an inquiry learning, videodisc course to learn the basics.

Disadvantages of interactive videodisc instruction. There have been two major obstacles to the use of videodisc technology in education. The first is cost, but this is dropping rapidly. A good interactive videodisc learning station will cost about $\$ 2500$ to $\$ 3000$, but should drop to about $\$ 1000$ in a few years. The second is acceptance by students, teachers, and administrators. Self-paced or individualized learning systems are seen by many teachers as a threat to their traditional role in the classroom (Ebner et al., 1984).

Young children (ages 9-12) appear to adapt quicker to learner-controlled instruction than adult learners (Cushall et al., 1987). Nevertheless, self-paced, self-controlled interactive material has enhanced achievement and satisfaction for adult learners (Merrill, 1980). Generally, some learners prefer and some do not prefer having control over their learning process and need training to break life-long patterns of being told what to learn and the pace at which they should learn (Milheim and Azbell, 1988).

Course design. Developing a videodisc presentation is most effectively accomplished by a team. Team members assume roles depending on their expertise. The best productions include active participation from the learner (Kearsley and Frost, 1985). Therefore, careful planning and design is es- sential in developing effective programs.

Because designing interactive video lessons is relatively new, educators must use sound learning principles in computer-based instruction (Bunderson, 1980; Caldwell, 1980; Gagne et al., 1981; O'Shea and Self, 1983; Smith and Boyce, 1984). As research information on computer-based interactive video becomes available, more concrete guidelines, which may be inherently different from present computer-based instruction, will be established.

Hardware. There are two basic formats for transferring slides, videotape, and audio to discs. University instructional media specialists can transfer slides and videotape clips to $16-\mathrm{mm}$ film, then create a 1 -inch helical master tape. The helical master can then be sent to a company for transfer to laser disc or to a company for transfer to an optical disc. Today, master 20-cm compact laser discs costs about $\$ 1500$, while optical discs cost about $\$ 1650$. Some companies will transfer all media directly to a master optical disc using their own processes. The laser disc is a one-time "write only" process; additions cannot be made later. Slide video segments can be added later to optical discs. Laser discs may be less expensive, depending on the university's policy of charging for instructional services time and facilities, but the author loses control of his/her original material while the company makes the disc.

The hardware to operate the videodisc usually consists of a laser disc player, a television monitor, and a microcomputer (Schwartz, 1987). The videodisc looks like a phonograph record. A $30.5-\mathrm{cm}$ disc can store about 55,000 slides or visual frames, 556 five-hundred-page textbooks or 5400 still frames plus $43 \mathrm{hr}$ of sound. Cost of this equipment has been reduced, but prices generally go hand-in-hand with quality and features, and range from $\$ 1500$ - $\$ 7500$ (Sales et al., 1986). The durability of the configured hardware enables the videodisc lesson to be presented in many different locations. Several manufacturers are producing single units that include all the hardware in one convenient package.

Compact Video Disc (CVD) is an analog/ digital hybrid capable of delivering interactive mixed-media. It is a true multi-media format, using an improved encoding format to store video, audio, and digital on the same CD-size disc. It substantially increases the amount of information, both digital and analog, that can be stored on an optical disc:

- 18,000 individually addressable video frames can be contained in the constant angular velocity (CAV), fully interactive mode (l-see access time)

- $18 \mathrm{~min}$ of full-screen motion video is supported in the continuous play constant linear velocity (CLV) mode; or

- $10 \mathrm{~min}$ of fully interactive CAV video, which permits still frames, fast motion, and slow motion without recourse to frame buffers

- Holds a gigabyte of data when used purely as a data disc 
- Holds $16 \mathrm{~kb}$ of data per video frame when an entire frame is devoted to storing digital data

- Frame-by-frame access to images, film, and sound

- File format compatible with High Sierra standards.

The video can be stored in long-play CLV mode or in the interactive CAV mode. The output is a full motion, full color, noncompressed video signal.

A CVD drive can technically play CVD discs as "well as CD-audio discs. An RS-232 interface is used for computer control and exchange of limited information. A SCSI interface will be able to rapidly transmit all digital data from CVD and CD-ROM discs. CVD is the medium for encoding all types of signals (i.e., analog or digital video, digital audio, digital data or programs).

CVD drives and players will make it possible to have one standard format for interactive video games, music videos, visual and textual databases, CD-Audio, and CD-ROMs.

Software. Software for linking inquiry learning courses to videodiscs is at the "Model T" stage of development. Hypertext was introduced as a new system for organizing information on a computer in 1986. Since 1986, several commercial programming (authoring) systems have been introduced including Guide, Hypercard, Mentor/Mac Video, and Supercard. All of these programs (some people are calling them languages and some authoring systems) are based on the hypertext concept and provide unlimited possibilities for structuring courses.

"Hypertext is an electronic system for organizing and presenting information that is not limited by the boundaries of a page or the binding of a book. Linking, referencing, backtracking, and querying are all possible with hypertext. In theory, at least, instead of forcing our thoughts to fit the sequential format that paper imposes, hypertext allows us to structure our reading and writing nonsequentially, to fit the way we think and what we are thinking about" (Eckhardt, 1987).

Guide and Hypercard are hypertext systems for personal computers and consequently differ in many ways from the original concept. Described by its publisher (OWL International; Bellevue, Wash.) as "Hypertext for the Macintosh," Guide was the first serious attempt to bring the concept of hypertext to a personal computer. Superficially, Hypercard looks like a word processor, file, and outliner, yet it is a whole new concept providing unlimited cross-referencing, and layering of "unseen" (Mellin, 1987), but readily accessible, information and rapid access to videodisc images.

Words or graphics within a document can be created as "buttons" or windows with Guide and Hypercard in the Macintosh system. Changes in the document reveal successively deeper layers of information when buttons are clicked.

Mentor and MacVideo by Edudisc Corporation (Nashville, Term.) are more advanced forms of hypertext software. Together these programs allow full authoring capability to produce ones own interactive videodisc course. The developed videodisc can contain graphics, charts, art, slide material, videotape clips, text, and multiple choice and fillin-the blank questions, all with interactive feedback.

Supercard builds on Hypercard's capabilities using Hypertalk language to access the full color display characteristics of Mac II computers. In addition, Supercard expands on Hypercard's windows, hot buttons, menu and database power tools, enhancing speed of operation and ease of use. Supercard will probably be the software of choice for CVD systems. Supercard is supposed to be available mid-1989 for less than $\$ 200$.

How our strategy would improve those tried elsewhere. Smith and Jones (1986). have effectively used computer-assisted videodiscs in teaching chemistry laboratory courses. However, the ratio of lesson preparation time to student use time was as high as 100:1. Not many professors are given the release time nor are willing to spend their weekends and evenings developing this type of course material.

We propose developing a consortium of professors at 12 to 15 universities, each preparing one or two lessons. The principal leader at Penn State will demonstrate how a lesson is developed for the videodisc format, collect the visual material, have it mastered on 1inch tape by instructional services personnel at Penn State, and transferred to CVD or laser discs. Sufficient copies of the discs will be made so that each university can use them for any number of applications.

The CVD format will provide a vastly larger potential beginning, post-education, and adult student audience. Eventually, it will be possible to upgrade most personal computers to accept the CVD players. The same players can be used for vast amounts of informational resources, including large libraries of visual art, stereo music, and literature search databases. Although computer-assisted videodisc teaching is only one component of the total proposed innovative delivery system, it is a vital link in providing self-paced learning to a heterogenous, geographically dispersed group of students. The horticulture courses would be disseminated by the various delivery systems described previously and become a demonstration of the new technology for all units of Penn State and for other land-grant universities in the United States.

\section{CONCLUSIONS}

Horticultural instruction can be informative, self-pacing, fun, and entertaining. The instructional delivery system can be efficient and cost-effective when inquiry learning and videodisc systems are used. Inquiry learning with videodiscs has great promise as an innovative teaching method for horticultural instruction. As educators, we are challenged to learn about new teaching delivery systems, such as interactive video, that provide the highest quality and most efficient education possible for our students.
Author's note. Because of the significant time commitment needed to develop an inquiry learning computer course, we are asking teachers of the basic or beginning courses in horticulture and plant identification to join with the faculty at Penn State who teach these courses in assembling a series of "National Standard" lectures. We are asking teachers of these courses to submit their course outlines to us for comparison and selection of common lecture elements. We will compile this information and send it back to all who submit outlines.

We will conduct an inquiry-learning video disc workshop at the ASHS Annual Meeting to be held at Penn State, 21-26 July 1991. The purposes of this workshop will be to: a) demonstrate state-of-the-art hardware and software using a sample lecture, b) ask teachers to help select a series of topics for which they would be willing to develop visuals and computer-based instructional material that could be put on videodiscs and shared with other teachers, c) provide basic training in development of computer-based instructional material.

The product of the shared outlines and workshop will be a videodisc or series of videodiscs that can be used by teachers everywhere to: a) supplement their present lectures, b) fill in gaps in their expertise, c) be used as part of audiotutorial sessions, or d) as stand-alone inquiry learning courses. If well-done and generally accepted, these lectures could form the basis for a minimum national standard of basic horticultural education.

\section{Literature Cited}

Bunderson, C.V. 1980. Instructional strategies for videodisc courseware: McGraw Hill Disc. J. Educ. Technol. Sys. 8:3.

Bunderson, C.V., B. Baillio, and J.B. Olsen. 1984 Instructional effectiveness of an intelligent videodisc in biology. Machine Mediated Learning 1(2): $175-215$

Caldwell, R. 1980. Guidelines for developing basic skills instructional materials for use with microcomputer technology. Educ. Technol. 20(10):712

Clark, D. 1984. How do interactive videodisc rate against other media? Instructional Innovator 29(6): 12-16.

Cohen, V. 1984. Interactive features in the design of videodisc materials. Educ. Technol. 24(1):1620.

Cushall, M. B., F.A. Harvey, and A.J. Brovey. 1987. Research on learning from interactive videodiscs: A review of the literature and suggestions for future research activities. Paper presented at Annual Conv. Assoc. Educ. Comm. and Technol 26 Feb.-1 Mar. 1987. Atlanta, Ga. (Ed. Res. Info. Center, Microfiche ED285530.)

Ebner, D., B. Danaher, J. Mahoney, L. Henry, and P. Balson. 1984. Current issues in interactive video and computer based instruction. Instructional Innovator 29(3):24-29.

Eckhardt, R.C. 1987. Glimpsing the future with guide. MacWorld 4(2):142-145.

Emmett, A. (asst. ed.). 1984. Discovering a new way to learn. Personal Computing. January. p. 56-189.

Fuller, R., D. Zollman, and T. Campbell. 1982. Videodiscs: The puzzle of the Tacoma Narrows 
Bridge collapse videodisc. Wiley, New York.

Gagne, R.M., W. Wagner, and A. Rojas. 1981. Planning and authoring computer-assisted instruction. Educ. Technol. 21(9):177-226.

Gindele, J.F. and G.H. Gindele. 1985. Interactive videodisc technology and its implications for education. Tech. Horizons in Educ. J. 12(1):9397.

Guskey, T.R. and J.Q. Easton. 1988. Improving student learning in college classrooms. Charles Thomas, New York.

Hannaway, D.B., P.J. Ballerstedt, P.E. Shuler, D.P. Connell, and D.N. Osterman. 1988. An interactive videodisc module for forage quality and testing instruction. J. Agron. Educ. 17:119121.

Kay, S. and T.G. Tate. 1986. The role of interactive video in agricultural education. USDA Washington, D.C. IVEN Working Paper 3.

Kearsley, G. and J. Frost. 1985. Design factors for successful video-based instruction. Educ. Technol. 25(3):7-13.

Malone, T.W. 1981. Toward a theory of intrinsically motivating instruction. Cognitive Sci. 4:333-369.
Mellin, C. 1987. A prototype science interactive videodisc: research on in-school use. Tech. Rpt. Apr. 1987. Office Educ. Res. and Improvement. Washington, D.C.

Merrill, M.D. 1980. Learner and control in computer based learning. Computers and Educ. 4:7795.

Milheim, W.D. and J.W. Azbell. 1988. How past research on learner control can aid in the design of interactive video materials. Proceedings of selected research papers at annual meeting of Assn. for Educ. Commun. and Technol. 14-19 Jan. 1988. New Orleans, La.

O'Shea, T. and J. Self. 1983. Learning and Teaching with Computers. Prentice-Hall, Englewood Cliffs, N.J.

Russell, A., G. Nugent, C. Stone, and R. Nugent. 1985. The use and evaluation of videodiscs in the chemistry laboratory. J. Chem. Educ. 62(5):420-422

Sales, G.C., J. Gordan, and R. Anderson. 1986. Videodisc design is easier and less expensive than you think. Tech Trends 31(5/6):30-35.

Schwartz, E. 1987. The educator's handbook to interactive videodisc. 2nd ed. Assn. for Educ. Commun. and Technol. Washington, D.C. (Ed. Res. Info Center, Microfiche Ed 283504.)

Smith, P.L. and B. Boyce. 1984. Instructional design considerations in the development of computer-assisted instruction. Educ. Technol. 24(7):5-16.

Smith, S.G. and L.L. Jones. 1986. The video laboratory a new element in teaching chemistry. Perspectives in Computing 6(2):20-26.

Solomon, G. 1983. Television watching and mental effort: A social psychological view, p. 181198. In: J. Bryant and D. Anderson (eds.). Children's understanding of television. Academic, New York.

Weiss, C. and S. Jarvis. 1986. The interactive videodisc for computer-assisted education and training in developing countries. Educ. Technol. Systems 15(1):3-19.

Wollenberg, J.P., H.M. Handley, and J.R. Enochs. 1985. Differences in achievement with computer-assisted instruction:implications for varying student learning styles. Educ. Technol. 25(11):51.

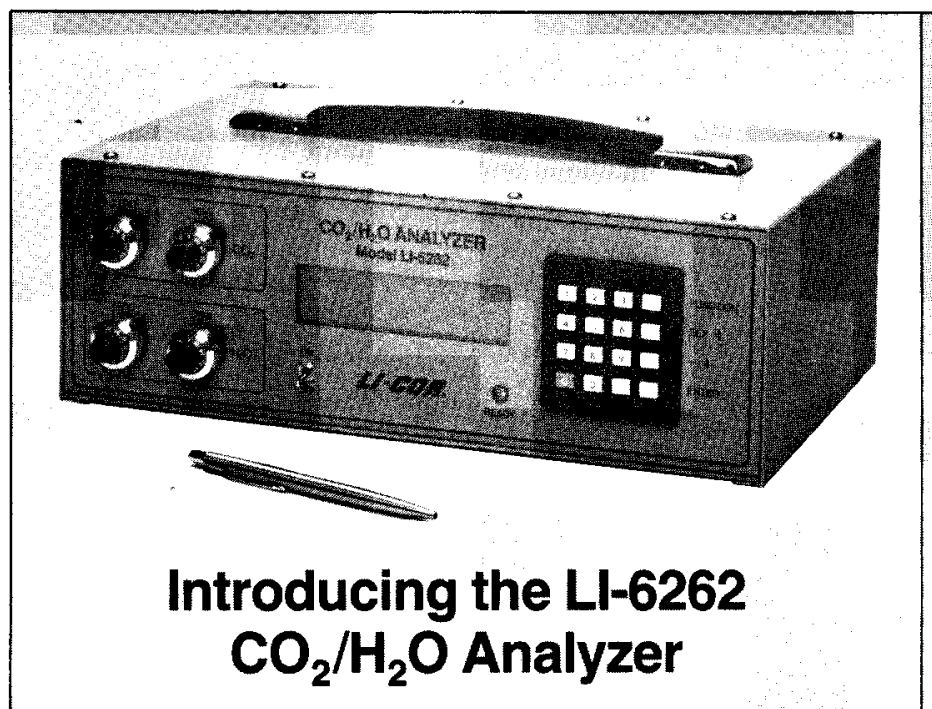

- Independent absolute or differential operation for $\mathrm{H}_{2} \mathrm{O}$ and $\mathrm{CO}_{2}$

- Digital readout in mole fraction, partial pressure or dewpoint $\left(\mathrm{H}_{2} \mathrm{O}\right)$

- RS-232C or linear analog output for data acquisition

- $\mathrm{CO}_{2}$ corrected for foreign gas broadening due to $\mathrm{H}_{2} \mathrm{O}$ vapor

\section{New Gas Analyzer for Simultaneous $\mathrm{CO}_{2}$ and $\mathrm{H}_{2} \mathrm{O}$ Measurements}

The LI-6262 is a differential, non-dispersive gas analyzer specially designed to make simultaneous $\mathrm{CO}_{2}$ and $\mathrm{H}_{2} \mathrm{O}$ measurements. The LI-6262 incorporates a modified $\mathrm{LI}-\mathrm{COR} \mathrm{CO}_{2}$ analyzer that provides high sensitivity, excellent stability and fast response for both $\mathrm{CO}_{2}$ and $\mathrm{H}_{2} \mathrm{O}$ measurements.

The LI-6262 is factory calibrated over a $0-3000$ ppm $\mathrm{CO}_{2}$ measurement range and has a typical signal noise level of only $0.2 \mathrm{ppm}$ peak-to-peak at $350 \mathrm{ppm}$. $\mathrm{H}_{2} \mathrm{O}$ calibrations are performed using a precisely controlled dewpoint generator and a dewpoint hygrometer. Typical signal noise levels for the $\mathrm{H}_{2} \mathrm{O}$ channel are $0.05 \mathrm{mB}$ peak-to-peak at $20 \mathrm{mB}$ vapor pressure.

For more information, call us at (402) 4673576. Or write: LLI-COR inc., P.O. Box 4425, Lincoln, Nebraska 68504, U.S.A.

FAX: 402-467-2819 TWX: 910-621-8116 